\title{
Germanica
}

\section{Écriture et plurilinguisme : « enrôlés dans la langue, arrivés en littérature »}

Schreiben und Mehrsprachigkeit: eingezogen in die Sprache, angekommen in der Literatur

\section{Nicole Bary}

\section{OpenEdition}

\section{Journals}

Édition électronique

URL : http://journals.openedition.org/germanica/618

DOI : $10.4000 /$ germanica. 618

ISSN : 2107-0784

\section{Éditeur}

Université de Lille

\section{Édition imprimée}

Date de publication : 1 juin 2009

Pagination : 123-130

ISBN : 978-2-913857-23-0

ISSN : 0984-2632

Référence électronique

Nicole Bary, «Écriture et plurilinguisme : «enrôlés dans la langue, arrivés en littérature » », Germanica [En ligne], 44 | 2009, mis en ligne le 01 juin 2011, consulté le 10 décembre 2020. URL : http:// journals.openedition.org/germanica/618; DOI : https://doi.org/10.4000/germanica.618

Ce document a été généré automatiquement le 10 décembre 2020.

(c) Tous droits réservés 


\section{Écriture et plurilinguisme : " enrôlés dans la langue, arrivés en littérature $»^{1}$}

Schreiben und Mehrsprachigkeit: eingezogen in die Sprache, angekommen in der Literatur

Nicole Bary

1 «Je ne suis qu'un hôte dans la langue allemande. L'allemand est une langue qui s'est implantée en moi tardivement, douloureusement. C'est pour cette raison qu'elle est devenue la langue de ma prose littéraire, parce qu'elle est toujours restée pour moi entourée d'une aura d'étrangeté qui charge les mots d'une passion tout à fait particulière » écrit Elias Canetti qui n'apprit l'allemand qu'à l'âge de huit ans. L'aura de l'étrangeté, la passion particulière que l'on peut éprouver à soumettre son écriture aux dangers, aux surprises, au bouillonnement qu'apporte «l'autre langue » sont-elles un aiguillon pour l'écriture, une sorte de plus value pour une littérature qui ne se nourrit que d'une seule culture?

2 À l'époque des retours identitaires, des déracinements, de l'effacement des frontières et du métissage des cultures et des langues, à l'époque de la cyberculture, on observe une grande mobilité des écrivains, une mobilité qui n'est pas nécessairement vécue comme un exil, ni comme une émigration politique, sociale, économique, mais comme un déplacement fait d'allées et venues, d'allers et de retours, de "diasporisations" transversales et plurielles.

3 Comme l'a analysé Pierre Bourdieu, la situation de l'écrivain dans cette mouvance du déplacement varie en fonction de l'histoire du pays qu'il quitte, des conditions dans lesquelles il le quitte, de l'histoire et de la situation politique du pays dans lequel il s'installe et de la spécificité des champs littéraires auxquels il est confronté, c'est-à-dire d'un ensemble institutionnel complexe d'écrivains qui se définissent par leur style, les formes qu'ils choisissent, le type d'histoires qu'ils racontent. Se crée-t-il ainsi «un langage qui utilise des poétiques peut-être opposées... des écritures de l'entre-deux, de la béance, 
de l'interstice, de l'enracinerrance $»^{2}$, des écritures du déplacement qui façonnent le nouvel imaginaire, et confèrent une autre dimension aux littératures dites « nationales »?

Deux remarques préliminaires: à la différence de la France ou de l'Angleterre, l'Allemagne n'a pas de tradition coloniale et donc pas d'écrivains germanophones issus de la colonisation. D'autre part les écrivains allemands qui ont quitté l'Allemagne à la suite de l'arrivée au pouvoir d'Hitler n'ont généralement pas changé de langue pendant leur exil. L'allemand était le lien qui les reliait au pays perdu, à la Heimat. Robert Neumann et Rose Ausländer ont certes tenté leur chance en anglais, mais peu satisfaits de leurs essais, ils sont revenus vers leur langue maternelle.

5 Au fil des cinquante dernières années, de nombreux écrivains venus d'ailleurs se sont fait connaitre dans les pays de langue allemande, d'abord des Turcs issus de l'émigration économique, et des exilés politiques (essentiellement en RFA) en provenance du monde entier, enfin depuis 1989 - et surtout à Berlin - de jeunes écrivains attirés par le dynamisme culturel de la capitale allemande. La langue allemande a fait preuve d'une grande imagination, et aussi d'une incroyable richesse, pour désigner la littérature de ceux qui, venus en Allemagne, ont troqué leur langue maternelle pour la langue nationale du pays où ils se sont installés ${ }^{3}$. Il a fallu des années pour que cette littérature - qui se présentait elle-même sous le nom de «Südwind Gastarbeiterdeutsch $»^{4}$ - soit reconnue comme une partie intégrante de la littérature allemande contemporaine. L'écrivain d'origine turque Zafer şenocak a depuis une vingtaine d'années mis en garde contre la dichotomie qui a longtemps divisé le champ littéraire allemand entre littérature de langue allemande patrimoniale et littérature étrangère en allemand, entre la langue comme héritage ou comme acquis. Cette dichotomie, dit-il, ne peut être dépassée que dans une confrontation des cultures allemandes du passé et du présent, non pas « une confrontation de la culture allemande avec la culture du pays d'origine.» Si dans les premières décennies la perte du pays d'origine (Heimat), l'arrivée difficile dans l'autre pays - qui malgré la terminologie en vigueur ne s'est pas révélé être un pays d'accueil, mais a fait perdurer chez le nouvel arrivant le sentiment qu'il est un étrange étranger - ont été les sujets les plus fréquemment traités, une rupture apparaît dans l'approche des thèmes, dans l'écriture, dans le choix de la langue au cours des deux dernières décennies du $\mathrm{xx}^{\mathrm{e}}$ siècle. La publication du premier roman d'Emine Sevgi Özdamar annonce un changement de paradigme. «Lorsque j'ai écrit mon premier roman ${ }^{5}$ ", écrit-elle, «je n'ai pas réfléchi dans quelle langue j'allais l'écrire. Le rythme de mon corps était allemand, mon quotidien était allemand, j'avais réalisé mon rêve, en allemand.» Özdamar, comédienne, est venue de Turquie en Allemagne dans les années quatre-vingt pour parfaire et élargir sa formation, attirée par le célèbre disciple de Brecht, Benno Besson, qui dirigeait à l'époque la Volksbühne de Berlin-Est. "Ma rencontre avec les mots allemands a eu lieu d'abord au théâtre » dit Özdamar qui poursuit « le théâtre est un dialogue entre les corps. Pour moi les mots allemands ont un corps ». La richesse narrative, le regard distancié et déroutant, la sensualité de la langue, le foisonnement des descriptions ne s'inscrivent pas dans une démarche introduisant dans le champ littéraire l'exotisme, mais dans la volonté de dire une autre expérience du réel et un autre imaginaire avec des mots allemands, de donner à ces mots une tonalité, une chair, une couleur, une lumière nouvelles. Il ne s'agit en aucun cas d'exotisme, mais d'une posture littéraire proche du concept de « créolisation » tel que l'a défini Édouard Glissant : la tentation d'échapper par des variations à la contrainte de la langue. 
C'est dans cette démarche que s'inscrit Saša Stanišić, dans son premier roman, Wie der Soldat das Grammophon reparierte ${ }^{6}$. Grand narrateur lui aussi, il redonne des lettres de noblesse à un genre un peu oublié, le roman picaresque et burlesque. Empruntant à cette tradition littéraire l'usage du long titre qui coiffe les différents chapitres, - usage qu'il partage avec chez Ingo Schulze - il structure ainsi le récit de son protagoniste, un jeune garçon qui vit à Višegrad en Bosnie et raconte sur le mode tragi-comique les mille et une aventures de sa famille avant et pendant la première guerre de Yougoslavie. Ces histoires retracent les différentes étapes de la destruction de Višegrad: au début du roman le lieu quasi idyllique d'une vie familiale animée et agitée, puis celui de la guerre, de l'horreur des combats et des massacres, de la violence, de la destruction, de la mort, enfin le point de départ vers l'exil. La famille du jeune Aleksander quitte la Bosnie en 1992, se réfugie en Allemagne. Dix ans plus tard le jeune homme retourne à Višegrad, pour retrouver une amie d'enfance : partout tristesse et désolation. Le jeune homme rencontre des membres de sa famille et des amis restés en Bosnie, certains acceptent de parler, d'autres non. Le refoulement de la mémoire est-il une grâce pour survivre? s'interroge en sourdine le narrateur. Le roman de Saša Stanišić est une sorte d'écho à celui d'Ivo Andric, Le Pont sur la Drina. Comme Andric, Stanisic veut dépasser la haine, contribuer à la réconciliation en revenant, sur le mode tragi-comique, sur les antagonismes historiques qui divisent le Sud-Est européen, non pas seulement pour apaiser la souffrance, mais pour éviter qu'elle ne se transforme en haine et en désir de revanche.

7 Avec Saša Stanišić, ce sont les divisions de l'Europe qui font irruption dans la littérature allemande. Né en 1978 à Višegrad, fils d'un Serbe et d'une Bosniaque, il a vécu l'assaut des troupes serbes sur sa ville. Le nationalisme a tué son identité, l'exil la lui a rendue, dit-il ${ }^{7}$. Il avait quatorze ans lorsque sa famille se réfugia en Allemagne. En quelques mois, il passa du serbe à l'allemand.

Il y a maintenant des centaines d'écrivains partout dans le monde qui, comme lui, ont choisi d'abandonner la langue de l'enfance, la langue de la filiation pour l'autre langue, celle du pays où ils résident, élargissant ainsi considérablement le champ des littératures nationales. Il faudrait toutefois se garder de donner à la différence culturelle une valeur ontologique ou essentialiste. Il semble plus intéressant de se demander dans quelle mesure la différence culturelle interroge les littératures nationales et ouvre de nouvelles perspectives esthétiques et poétiques transculturelles et post-nationales. Dans un essai ${ }^{8}$, Saša Stanišić raconte son étonnement lorsqu'il lut sous la plume d'un critique allemand «Stanišic met notre vieil allemand sous une tente à oxygène ». Il est évidemment possible, dit-il, que la pénétration de l'autre langue donne des résultats excellents, on traduit littéralement la langue d'où l'on vient, on lui emprunte des tournures et des expressions, on distord ses structures syntaxiques, on imite ses rythmes, on se risque même à quelques néologismes. Mais interroge-t-il, l'écriture n'est-elle pas toujours appropriation de rythmes, de structures, de mots auxquels l'écrivain donne sa propre tonalité? Le terrain de l'expérimentation, l'emprunt aux autres langues et cultures sont-ils réservés aux seuls écrivains qui viennent d'ailleurs alors qu'ils seraient interdits aux écrivains issus de la littérature dite nationale? « La langue, rappelle-t-il, est le seul pays sans frontière. Tout le monde peut (et devrait) user de ce privilège pour rendre une langue plus grande, meilleure et plus belle, en plantant un arbre-mot qui n'existe pas encore. » 
Qu'apporte à la littérature la confrontation des langues? Dans son essai In jeder Sprache sitzen andere Augen ${ }^{9}$, Herta Müller pose clairement la question de la confrontation des langues dans l'imaginaire de l'écrivain bilingue : «Dans la langue de mon village - c'est du moins l'impression que j'avais quand j'étais enfant - pour tous les gens de mon entourage les mots correspondaient aux choses qu'ils désignaient. Les choses s'appelaient exactement comme elles étaient, et elles étaient exactement comme elles s'appelaient. Une adéquation claire une fois pour toutes. Pour la plupart des gens il n'y avait entre les mots et les choses aucun espace au travers duquel on aurait pu risquer un regard et fixer le néant. » Une adéquation parfaite des mots aux choses? Lorsque Herta Müller germanophone de naissance quitte son village du Banat pour le lycée et la ville, le roumain, la langue officielle du pays devient la langue du quotidien et elle découvre qu'à une même chose correspondent plusieurs dénominations. L'absolu de la langue maternelle est relativisée, d'autant plus que la nouvelle langue, le roumain, offre une palette d'images nouvelles, plus concrètes que l'allemand. Le lys, féminin en allemand est masculin en roumain comme en français. Et certainement, poursuit Herta Müller, « la » lys, la dame-lys regarde-t-elle autrement que le monsieur-lys. Roumaine de langue allemande originaire du Banat, Herta Müller joue dans ses textes de fiction avec les différentes appréhensions du monde que lui offrent les deux langues. Dans son écriture l'allemand et le roumain se conjuguent, ouvrant un espace entre les langues et les mots qu'investit l'écriture poétique.

10 C'est de cet espace, de cet entre-deux, de ce "dazwischen ", de cet entre-deux des langues, des cultures, des pays, des mémoires, des perceptions que langues, cultures, écritures se confrontent, se fécondent réciproquement, s'hybrident. C'est dans cet espace que prend forme le sentiment que le monde est plus grand, qu'il est pluriel, un «Mehrweltgefühl » écrit Marica Bodrožić. Née sur la côte dalmate qu'elle quitta à l'âge de dix ans pour l'Allemagne, elle explore dans son écriture tous les possibles interculturels. "Tous ceux qui parlent plusieurs langues savent que cette expérience est là en permanence, tout comme ils savent depuis Rimbaud que le «je » ne peut être saisi que dans la pluralité de ses acceptions ${ }^{10} »$.

11 Dans ses essais Sterne erben, Sterne färben, une véritable déclaration d'amour à la langue allemande, elle explique qu'en ce qui la concerne, écrire dans l'autre langue lui a donné, à elle qui est bilingue, la possibilité de forger sa propre langue littéraire, de trouver son écriture. Sa langue maternelle, le serbo-croate,très chargée émotionnellement ne pouvait pas, dit-elle, avoir cette fonction, il lui manquait un filtre. L'allemand le lui a apporté. "L'allemand possède une telle gamme de rythmes, de tonalités, que quelquefois la tête me tourne quand j'écris, car j'entends alors la musique de la langue, je suis cette musique en écrivant, je l'écoute en moi, le long des mots ${ }^{11}$ », une définition de l'identité poétique que ne démentirait pas Ilma Rakusa.

Née en Slovaquie, d'une mère hongroise et d'un père slovène, elle a grandi successivement en Slovaquie, en Hongrie, en Slovénie, à Trieste avant que ses parents s'installent à Zurich avec elle. "Mon oreille était sensible aux langues : elles étaient signe de richesse et de différences. Ce que je ne comprenais pas, m'inspirait une sensation de malaise et éveillait en moi le désir de surmonter cette étrangeté en apprenant la langue inconnue. J'avais six ans lorsque nous sommes allés nous installer à Zurich et l'allemand s'est ajouté aux autres langues. L'allemand devint pour moi la langue la plus importante : la langue de mes textes littéraires. C'est seulement en allemand que je peux exprimer les nuances stylistiques les plus fines, et si je dialogue 
avec moi-même, c'est le plus souvent en allemand. Il n'y a pas de doute : je suis un écrivain de langue allemande, avec, évidemment, une préhistoire insolite ${ }^{12}$ ». Une telle biographie suppose de nombreux passages de frontières et une expérience à chaque fois renouvelée que l'herbe n'est pas plus verte, ni les arbres plus beaux de l'autre côté de la frontière. L'œil ne saisit pas les différences que l'oreille perçoit, L'étranger ne commence pas seulement à la barrière de la frontière, l'étranger commence dès qu'on ne comprend plus la langue, dès que l'autre langue frappe à l'oreille. C'est sur cette expérience que se construit la conscience de l'altérité et le désir de transgression, c'est là que s'articulent les rapports de force entre l'un et le multiple, l'intérieur et l'extérieur, le familier et l'étranger, le proche et le lointain. C'est sur cette expérience que se fonde le désir d'explorer le territoire où ces forces contradictoires s'affrontent, le désir d'être un passeur entre des extrêmes, le désir d'écrire : "L'étranger dont je parle ne m'est pas extérieur, il est une partie de moi, un extérieur intériorisé qui m’a conduit à dire, en paraphrasant le «Je est un autre» de Rimbaud, «Je est multiple». Voilà une définition de l'identité poétique $»^{13}$.

Ne pas s'enraciner ce n'est pas ne pas avoir de racines. Bizarrement cela signifie

être mobile [...] Prendre racine dans l'itinérance ${ }^{14}$.

C'est dans l'œuvre de Yoko Tawada, particulièrement dans Wo Europa anfängt ${ }^{15}$ ? que l'on trouve une véritable poétique du déplacement, du franchissement de frontière, de la mobilité et de la différence culturelle comme préalables à l'écriture. Le texte de Yoko Tawada renvoie à l'expérience de l'auteur dans son voyage transculturel - en bateau, puis avec le Transsibérien, du Japon à Moscou - Il n'a pas l'intention d'être un récit de voyage, il est composé d'éléments hétérogènes qui mêlent souvenirs du pays que l'on quitte, contes, récits - oniriques parfois - évocations des contrées parcourues et réflexions intérieures. Yoko Tawada puise dans le fonds mondial des mythes et légendes et les mêle savamment. Dans le premier récit une jeune fille sur les conseils d'un serpent (féminin en allemand) part vers une ville lointaine avec sa mère gravement malade pour chercher auprès d'un oiseau de feu la guérison. Mais arrivée au but du voyage, elle oublie les conseils du serpent, boit de l'eau de la ville, se transforme en une vieille femme et perd sa mère. Relation mère-fille, relation parents-enfants, opposition de l'eau et du feu sont les thèmes repris dans les textes suivants dans de multiples variations - au sens musical du terme. À l'arrivée à Moscou, la narratrice voit une inscription en lettres de feu au-dessus de la ville dont la lettre A se transforme en une pomme, Apfel en allemand, dans laquelle elle mord: la mère et tout ce qui, dans les différents récits, se rattache à elle, donc au passé disparaissent définitivement et marquent l'arrivée en Europe. Avant de goûter à la pomme de la connaissance, la narratrice a traversé les grands espaces de Sibérie à la recherche de frontières, une quête que le titre laisse deviner. Les connotations littéraires en liaison avec le but du voyage - Moscou - et la Sibérie perçue comme un espace de transit autorisent à lire le texte comme une référence personnelle de l'auteur à un voyage initiatique et transculturel.

Née à Tokyo en 1960, Yoko Tawada vit en Europe depuis 1982. Écrivain à l'identité double, elle écrit dans sa langue maternelle et en allemand. Elle s'en explique: «Lorsque j'étais enfant, j'ai su très vite que j'écrirais, j'écris depuis l'âge de douze ans. J'ai su très vite que le japonais n'était pas une langue suffisante pour écrire. Il me fallait une autre langue. La russe était la plus belle des langues pour écrire, ensuite venait le français et l'allemand à cause de Kafka... À l'époque les Russes n'acceptaient pas d'étudiants japonais, j'ai dû renoncer à la Russie. J'aimais le français, mais mes 
professeurs n'étaient pas assez indulgents, ils ne me pardonnaient pas mes erreurs. Ce sont pourtant les erreurs qui produisent la littérature. Va pour l'allemand! $»^{16}$

Dans ses écrits suivants, Yoko Tawada a poursuivi sa réflexion théorique sur l'identité poétique et culturelle, en particulier dans ses essais Von der Muttersprache zur Sprachmutter ${ }^{17}$ où elle thématise avec humour les expériences d'une jeune femme tout juste arrivée du Japon qui transforme le travail quotidien dans un bureau allemand en une succession de postures énigmatiques. Ce sont surtout les genres des mots qui la désespèrent, ils restent étrangers à l'objet. Dans un univers d'objets essentiellement masculins, le seul refuge féminin est la machine à écrire qui devient une mère de substitution: "Elle avait un large corps tatoué, imposant sur lequel on pouvait lire toutes les lettres de l'alphabet. Lorsque je m'asseyais devant elle, j'avais l'impression qu'elle m'offrait un langage. Sa proposition ne changeait pourtant rien au fait que l'allemand n'était pas ma langue maternelle, mais en échange la machine devint une mère-langue. » Grâce à l'apparition d'une nouvelle mère, la narratrice peut vivre une seconde enfance : "Quand on a une nouvelle mère-langue, on peut vivre une nouvelle enfance. Quand on est enfant, on prend la langue au pied de la lettre. C'est ainsi que chaque mot a sa propre vie qui s'émancipe du sens de la phrase.»

Dans les textes plus récents de Yoko Tawada, le jeu avec les deux langues est l'une des nombreuses stratégies d'émancipation poétique qui se cachent derrière le langage faussement naïf de l'auteur.

L'irruption d'écrivains qui n'ont pas hérité de la langue allemande, mais l'ont choisie fait éclater les parois trop hermétiquement closes de la littérature allemande, au sens politique du mot. Ces écrivains ont vécu une autre histoire, leurs repères culturels, littéraires sont en Allemagne et hors d'Allemagne. L'histoire allemande récente, celle de la division et de la réunification, à fortiori celle du nazisme et de la culpabilité allemande n'est inscrite ni dans leur biographie, ni dans celle de leur famille. Leur histoire est celle du déplacement, du changement de perspective et de la relativité de toute perspective. Reste à savoir si ces différents versants de la littérature allemande contemporaine vont continuer à vivre, à se développer parallèlement ou s'ils vont se féconder réciproquement. Peut-être si, de part et d'autre, on apprend à penser l'hétéronomie, si, comme l'écrit Régine Robin, on apprend à penser "la noncoïncidence de soi à soi, de l'écart entre le territoire, la culture, la langue, et les modalités multiples de l'identité ».

\section{NOTES}

1. Positionen des Schreibens in unserem Einwanderungsland. Eingezogen in die Sprache, angenommen in der Literatur, Wallstein 2008, Valerio 2008/8.

2. Édouard Glissant, Le discours antillais, Le Seuil, 1981.

3. Gastarbeiterliteratur, Gastliteratur, Migrantenliteratur, Emigrantenliteratur, Literatur der Betroffenen, Brückenliteratur, Ausländerliteratur.

4. Lenke von Saalfeld, In den Stromschnellen der Sprache in Valerio 2008/8. 
5. Mutterzunge, Kiepenheuer \&Witsch, 1990.

6. Luchterhand Literaturverlag, 2006.

7. Entretien dans Télérama 3065.

8. Wie ihr uns seht. Über drei Mythen vom Schreiben der Migranten. In Valerio 8/2008.

9. In Der König verneigt sich und tötet, Hanser, 2003.

10. In Valerio 2008/8 Die Sprechländer des Dazwischen.

11. Sterne färben, Meine Ankunft in Wörtern. Suhrkamp 2007.

12. In Ilma Rakusa Zur Sprache gehen. Thelem 2006, p. 7 sq.

13. Ilma Rakusa, cf. note 10.

14. Ulrike Draesner, cité par Ilma Rakusa.

15. Konkursbuch/Verlag Claudia Gehrke 1991.

16. In Sprachpolizei und Spielpolyglotte, Konkursbuch/Verlag Claudia Gehrke, 2007.

17. Cf. note 14 .

\section{RÉSUMÉS}

À l'époque des retours identitaires, des déracinements, de l'effacement des frontières et du métissage des cultures et des langues, à l'époque de la cyberculture, on observe une grande mobilité des écrivains, une mobilité qui n'est pas nécessairement vécue comme un exil, ni comme une émigration politique, sociale, économique, mais comme un déplacement fait d'allées et venues, d'allers et de retours, de « diasporisations » transversales et plurielles. Comme l'a analysé Pierre Bourdieu, la situation de l'écrivain dans cette mouvance du déplacement varie en fonction de l'histoire du pays qu'il quitte, des conditions dans lesquelles il le quitte, de l'histoire et de la situation politique du pays dans lequel il s'installe et de la spécificité des champs littéraires auxquels il est confronté, c'est-à-dire d'un ensemble institutionnel complexe d'écrivains qui se définissent par leur style, les formes qu'ils choisissent, le type d'histoires qu'ils racontent. Emine Sevgi Özdamar, Sasa Stanisic, Ilma Rakusa et Yoko Tawada ont créé « un langage qui utilise des poétiques peut-être opposées... des écritures de l'entre-deux, de la béance, de l'interstice, de l'enracinerrance » (Édouard Glissand), des écritures du déplacement qui façonnent le nouvel imaginaire, et confèrent une autre dimension aux littératures dites « nationales ».

Im Zeitalter der Rückkehr zur Identität, der Entwurzelung, der Auflösung von Grenzen, der Vermischung von Kulturen und Sprachen, ja im Zeitalter der Cyberkultur weisen Schriftsteller innen eine hohe Mobilität auf, die nicht zwangsläufig als Exil oder politische, soziale und ökonomische Emigration erlebt wird, sondern als eine Bewegung aus Kommen und Gehen, Abschied und Heimkehr, wechselseitigen und vielfältigen «Diasporisierungen». Pierre Bourdieu zufolge ist die Situation der Schriftsteller innen bei diesen Ortswechseln abhängig von der Geschichte des Landes, das sie verlassen, von den Bedingungen, unter denen sie es verlassen, von der Geschichte und politischen Situation des Ziellandes und von den Besonderheiten des literarischen Felds, auf das sie stoßen. Dieses ist wiederum ein komplexes institutionelles Ensemble aus Schriftsteller innen, die sich durch ihren Stil, die von ihnen gewählten Formen und die Art der Geschichte, die sie erzählen, definieren. Emine Sevgi Özdamar, Saša Stanišić, Ilma Rakusa und Yoko Tawada haben «eine Sprache [erfunden], die potenziell gegensätzliche Poetiken verwendet, ein Schreiben des Dazwischen, der Lücke, des Zwischenraums und der Verentwurzelung» (Édouard Glissant), also ein Schreiben der Bewegung, das eine neue 
Imagination formt und den so genannten «Nationalliteraturen» eine andere Dimension hinzufügt.

INDEX

Mots-clés : jeunes auteurs, nouvelle écriture, nouvelle génération d'auteurs

\section{AUTEURS}

NICOLE BARY

Traductrice

Directrice de la Bibliothèque allemande aux éditions Métailé et de l'association « Les Amis du Roi des Aulnes » 